\title{
On Cayley Graphs of Abelian Groups
}

\section{CAI HENG LI}

li@maths.uwa.edu.au

Department of Mathematics, University of Western Australia, Nedlands, W.A. 6907, Australia

Received March 9, 1995; Revised April 18, 1997

\begin{abstract}
Let $G$ be a finite Abelian group and $\operatorname{Cay}(G, S)$ the Cayley (di)-graph of $G$ with respect to $S$, and let $A=\operatorname{Aut} \operatorname{Cay}(G, S)$ and $A_{1}$ the stabilizer of 1 in $A$. In this paper, we first prove that if $A_{1}$ is unfaithful on $S$ then $S$ contains a coset of some nontrivial subgroup of $G$, and then characterize Cay $(G, S)$ if $A_{1}^{S}$ contains the alternating group on $S$. Finally, we precisely determine all $m$-DCI $p$-groups for $2 \leq m \leq p+1$, where $p$ is a prime.
\end{abstract}

Keywords: Cayley graph, isomorphism, CI-subset, $m$-DCI group

\section{Introduction}

Let $G$ be a finite group and $S$ a Cayley subset of $G$, that is, $S$ does not contain the identity of $G$. The Cayley (di)-graph $\operatorname{Cay}(G, S)$ of $G$ with respect to $S$ has the elements of $G$ as vertices and the pairs $(g, s g), g \in G, s \in S$, as edges. Given a Cayley subset $S$ of $G$, if, for any Cayley subset $T$ of $G, \operatorname{Cay}(G, S) \cong \operatorname{Cay}(G, T)$ implies $T=S^{\sigma}$ for some $\sigma \in \operatorname{Aut}(G)$, then $S$ is called a CI-subset (CI stands for Cayley Isomorphism). A finite group $G$ is called an $m$-DCI group if all of its Cayley subsets of $G$ of size at most $m$ are CI-subsets; $G$ is called a DCI-group if it is a $|G|$-DCI group. Similarly, $G$ is called an $m$-CI group if all Cayley subsets $S$ of $G$ of size at most $m$ with $S=S^{-1}$ are CI-subsets, $G$ is called a $C I$-group if $G$ is an $|G|-C I$ group. The problem of determining which groups are $m$-DCI groups and $m$-CI groups has been investigated for a long time, see $[6,10,12]$ for references. Recently, all $m$-DCI groups and all $m$-CI groups for $m \geq 2$ have been classified in [10] and [9], respectively, in the sense that all the possibilities for such groups are explicitly listed. However, it is still a difficult question to determine which of them are really $m$-DCI ( $m$-CI) groups. Babai and Frankl [2] asked whether the elementary abelian group $Z_{p}^{d}$ for any $p$ and $d$ was an $m$-CI group for all $m \leq|G|$ (in other words, $Z_{p}^{d}$ is a CI-group). Godsil [6] and Dobson [4] proved this to be true for $d=2,3$, respectively. However, recently Nowitz [11] gave a negative answer to the question by proving that $Z_{2}^{6}$ is not a 31-CI group. It is not known if this the answer of the question is positive for odd prime $p$ and $d \geq 4$. The main aims of this paper are to characterize Cayley graphs $\operatorname{Cay}(G, S)$ of abelian groups by the action of $A_{1}$ on $S$, where $A_{1}$ is the stabilizer of 1 in $\operatorname{Aut} \operatorname{Cay}(G, S)$, and to determine precisely $m$-DCI $p$-groups for $2 \leq m \leq p+1$, which implies that the answer of Babai and Frankl's question is positive for any $p, d$ and $m \leq p+1$.

Notation In this paper, $Z_{n}$ denotes a cyclic group of order $n, Q_{8}$ is the quaternion group of order 8. Recall that a group is called homocylic if it is a direct product of some cyclic 
groups of the same order. For groups $G$ and $H, H \leq G$ denotes that $H$ is a subgroup of $G$, and $G \rtimes H$ denotes a semidirect product of $G$ by $H$. For a positive integer $n, C_{n}$ denotes the directed cycle of length $n, K_{n}$ denotes the complete graph on $n$ vertices and $K_{n, n}$ denotes the complete-bipartite graph on $2 n$ vertices. For a directed graph $\Gamma=(V, E)$, its complement $\bar{\Gamma}=(V, \bar{E})$ is the directed graph with vertex set $V$ such that $(a, b) \in \bar{E}$ if and only if $(a, b) \notin E$. The direct product $\Gamma_{1} \times \Gamma_{2}$ of two directed graphs $\Gamma_{1}=\left(V_{1}, E_{1}\right)$ and $\Gamma_{2}=\left(V_{2}, E_{2}\right)$ is the directed graph with vertex set $V_{1} \times V_{2}$ such that $\left(\left(a_{1}, a_{2}\right),\left(b_{1}, b_{2}\right)\right)$ is an edge if and only if either $\left(a_{1}, b_{1}\right) \in E_{1}$ and $a_{2}=b_{2}$, or $\left(a_{2}, b_{2}\right) \in E_{2}$ and $a_{1}=b_{1}$. The lexicographic product $\Gamma_{1}\left[\Gamma_{2}\right]$ of two directed graphs $\Gamma_{1}=\left(V_{1}, E_{1}\right)$ and $\Gamma_{2}=\left(V_{2}, E_{2}\right)$ is the graph with vertex set $V_{1} \times V_{2}$ such that $\left(\left(a_{1}, a_{2}\right),\left(b_{1}, b_{2}\right)\right)$ is an edge if and only if either $\left(a_{1}, b_{1}\right) \in E_{1}$ or $a_{1}=b_{1}$ and $\left(a_{2}, b_{2}\right) \in E_{2}$. For any vertex $x$ of $\operatorname{graph} \operatorname{Cay}(G, S)$, the neighborhood $\Gamma(x)$ of $x$ in $\operatorname{Cay}(G, S)$ equals $x S=\left\{x a_{i} \mid 1 \leq i \leq m\right\}$. Let $\Gamma_{i}(x)=$ $\{y \in G \mid d(x, y)=i\}$, where $d(x, y)$ denotes the distance from $x$ to $y$ in $\operatorname{Cay}(G, S)$. Note that $\Gamma(x)=\Gamma_{1}(x)$.

In Section 2, we quote some results which are used in the following sections. Section 3 characterizes some Cayley graphs on Abelian groups, and Section 4 precisely determines $m$-DCI $p$-groups for certain values of $m$.

\section{Preliminaries}

In this section, we quote some results which we need in the following sections. Let $G$ be a finite group, $S$ a Cayley subset of $G$ and let $A=\operatorname{Aut} \operatorname{Cay}(G, S)$. Babai [1] gave a criterion for a subset of $G$ to be a CI-subset.

Theorem 2.1 ([1]) For a given group $G$ and a Cayley subset $S$ of $G, S$ is a CI-subset if and only if for any $\tau \in \operatorname{Sym}(G)$ with $\tau G \tau^{-1} \leq A$, there exists $\alpha \in A$ such that $\alpha G \alpha^{-1}=$ $\tau G \tau^{-1}$, where $\operatorname{Sym}(G)$ is the symmetric group on $G$.

The normalizer of $G$ in $A$ is often useful for characterizing $\operatorname{Cay}(G, S)$.

Lemma 2.2 ([5]) Let $A=\operatorname{Aut} \operatorname{Cay}(G, S)$ and $\operatorname{Aut}(G, S)=\left\{\alpha \in \operatorname{Aut}(G) \mid S^{\alpha}=S\right\}$. Then $\mathrm{N}_{A}(G)$ equals a semidirect product of $G$ by $\operatorname{Aut}(G, S)$, that is, $N_{A}(G)=G \rtimes A u t(G, S)$.

All finite $m$-DCI groups for $m \geq 2$ have been explicitly listed in [10], in particular, we have

Lemma 2.3 ([10, Proposition 3.1]) Let $G$ be a finite $m$-DCI $p$-group, where $m \geq 2$ and $p$ is a prime.

(1) If $p$ is odd and $2 \leq m \leq p-1$, then $G$ is homocyclic.

(2) If $m=p$, then either $G$ is elementary Abelian, cyclic, or $G=Q_{8}$.

(3) If $m=p+1$, then either $G$ is elementary Abelian, or $G=Z_{4}$ or $Q_{8}$.

Lemma 2.4 ([16]) The quaternion group $Q_{8}$ is a DCI-group. 


\section{Cayley graphs of Abelian groups}

In this section, we characterize some properties of Cayley graphs of Abelian groups. Let $G$ be a finite group, $S=\left\{a_{1}, a_{2}, \ldots, a_{m}\right\}$ be a Cayley subset of $G$ and $\Gamma=\operatorname{Cay}(G, S)$. Let $A$ be the full automorphism group of $\Gamma$ and $A_{1}$ the stabilizer of 1 in $A$. For $h$ distinct elements $a_{i_{1}}, a_{i_{2}}, \ldots, a_{i_{h}} \in S$ and $y \in G$, let

$$
\left\{\begin{array}{l}
\Gamma\left(y a_{i_{1}}, \ldots, y a_{i_{h}}\right)=\Gamma\left(y a_{i_{1}}\right) \cap \cdots \cap \Gamma\left(y a_{i_{h}}\right) \\
\Gamma^{*}\left(y a_{i_{1}}, \ldots, y a_{i_{h}}\right)=\Gamma\left(y a_{i_{1}}, \ldots, y a_{i_{h}}\right) \backslash \bigcup_{x \in R} \Gamma(y x),
\end{array}\right.
$$

where $R=S \backslash\left\{a_{i_{1}}, \ldots, a_{i_{h}}\right\}$, that is, $\Gamma^{*}\left(y a_{i_{1}}, \ldots, y a_{i_{h}}\right)$ is the set of all vertices of $\Gamma$ which are joined to every element of $\left\{y a_{i_{1}}, \ldots, y a_{i_{h}}\right\}$ and to no element of $y R$. Let

$$
\Gamma_{i}^{*}=\max \left\{\left|\Gamma^{*}\left(u_{1}, \ldots, u_{i}\right)\right| \mid u_{1}, \ldots, u_{i} \in S\right\}
$$

If $R=\left\{u_{1}, \ldots, u_{i}\right) \subseteq S$, then denote $\Gamma^{*}\left(u_{1}, \ldots, u_{i}\right)$ by $\Gamma^{*}(R)$ sometimes.

Lemma 3.1 Suppose that $G$ is an Abelian group. Then

(i) $1 \in \Gamma^{*}(W)$ for $W \subseteq S$ if and only if $W=W^{-1}$ and $(S \backslash W) \cap(S \backslash W)^{-1}=\emptyset$;

(ii) $\Gamma^{*}\left(x x_{1}, \ldots, x x_{k}\right)=x \Gamma^{*}\left(x_{1}, \ldots, x_{k}\right)$ for any $x \in G$ and any $x_{1}, \ldots, x_{k} \in S$;

(iii) $\Gamma_{k}^{*} \leq k$ for every $k \geq 1$;

(iv) every element of $\Gamma_{2}(1)$ lies in $\Gamma^{*}\left(x_{1}, \ldots, x_{k}\right)$ for some $x_{1}, \ldots, x_{k} \in S$.

Proof: By the definition of $\Gamma^{*}\left(x_{1}, \ldots, x_{k}\right)$, part (i) is clear. Again by definition, we have

$$
\begin{gathered}
y \in \Gamma^{*}\left(x x_{1}, \ldots, x x_{k}\right) \Leftrightarrow y \in \Gamma^{*}\left(x x_{1}\right) \cap \cdots \cap \Gamma^{*}\left(x x_{k}\right) \backslash \bigcup_{z \in R} \Gamma(x z) \\
\Leftrightarrow x^{-1} y \in \Gamma\left(x_{1}\right) \cap \cdots \cap \Gamma\left(x_{k}\right) \backslash \bigcup_{z \in R} \Gamma(z) \\
\Leftrightarrow y \in x\left(\Gamma\left(x_{1}\right) \cap \cdots \cap \Gamma\left(x_{k}\right) \backslash \bigcup_{z \in R} \Gamma(z)\right) \\
=x \Gamma^{*}\left(x_{1}, \ldots, x_{k}\right),
\end{gathered}
$$

where $R=S \backslash\left\{x_{1}, \ldots, x_{k}\right\}$. Thus part (ii) is true. Now suppose that $\Gamma_{k}^{*}=\left|\Gamma^{*}\left(x_{1}, \ldots, x_{k}\right)\right|$ for some $x_{1}, \ldots, x_{k} \in S$. By definition, $x_{1} x \notin \Gamma^{*}\left(x_{1}, \ldots, x_{k}\right)$ for any $x \in S \backslash\left\{x_{1}, \ldots, x_{k}\right)$, so $\Gamma^{*}\left(x_{1}, \ldots, x_{k}\right) \subseteq\left\{x_{1} x_{1}, \ldots, x_{1} x_{k}\right\}$. Hence $\Gamma_{k}^{*}=\left|\Gamma^{*}\left(x_{1}, \ldots, x_{k}\right)\right| \leq k$ as in (iii). Finally, for any $y \in \Gamma_{2}(1)$, let $\left\{x_{1}, \ldots, x_{k}\right\}=\{x \in S \mid y \in \Gamma(x)\}$. Then $y \in \Gamma^{*}\left(x_{1}, \ldots, x_{k}\right)$ is as in (iv).

It is clear that if $\operatorname{Cay}(G, S) \cong C_{l}\left[\bar{K}_{m}\right]$ for $m>1$ then $A_{1}$ is not faithful on $S$. Conversely, the following theorem shows that if $A_{1}$ is not faithful on $S$ then $\operatorname{Cay}(G, S)$ contains such a subgraph. 
Theorem 3.2 Let $G$ be an Abelian group and $\Gamma=\operatorname{Cay}(G, S)$ for some $S \subset G$ such that $G=\langle S\rangle$. Let $A=$ Aut $\Gamma$ and $A_{1}$ the stabilizer of 1 in $A$. Then either $A_{1}$ is faithful on $S$, or $S$ contains a coset of some nontrivial subgroup of $G$ and $\Gamma$ has a subgraph isomorphic to $C_{l}\left[\bar{K}_{n}\right]$ for some integers $l$ and $n$.

Proof: Let $S=\left\{a_{1}, a_{2}, \ldots, a_{m}\right\}$. Assume first that for any integer $h \geq 1$ and any $h$ elements $x_{1}, \ldots, x_{h} \in S,\left|\Gamma^{*}\left(x_{1}, \ldots, x_{h}\right)\right| \leq 1$. We claim that $A_{1}$ is faithful on $S$. For any $y \in \Gamma_{2}(1)$, let $\left\{a_{i_{1}}, \ldots, a_{i_{h}}\right\}=\{x \in S \mid y \in \Gamma(x)\}$. Then $y$ is the unique element of $\Gamma^{*}\left(a_{i_{1}}, \ldots, a_{i_{h}}\right)$. If $\alpha \in A_{1}$ such that $x^{\alpha}=x$ for all $x \in S$, then $\alpha$ fixes $a_{i_{1}}, \ldots, a_{i_{h}}$. Thus $\alpha$ fixes $\Gamma^{*}\left(a_{i_{1}}, \ldots, a_{i_{h}}\right)$, and so $\alpha$ fixes $y$. Hence $x^{\alpha}=x$ for all $x \in \Gamma_{2}(1)$. Since $\langle S\rangle=G$, $\operatorname{Cay}(G, S)$ is connected, and it follows that $x^{\alpha}=x$ for all $x \in V \Gamma$. Hence $\alpha=1$ and $A_{1}$ is faithful on $S$.

Assume now that there are some $h$ vertices $a_{i_{1}}, \ldots, a_{i_{h}}$ such that $\left|\Gamma^{*}\left(a_{i_{1}}, \ldots, a_{i_{h}}\right)\right| \geq 2$. Let $w, y \in \Gamma^{*}\left(a_{i_{1}}, \ldots, a_{i_{h}}\right)$. Without loss of generality, we may assume that $\left\{i_{1}, \ldots, i_{h}\right\}=$ $\{1, \ldots, h\}$. By the definition of $\Gamma^{*}\left(a_{1}, \ldots, a_{h}\right)$, there exist $u_{1}, \ldots, u_{h}, v_{1}, \ldots, v_{h} \in$ $\left\{a_{1}, \ldots, a_{h}\right\}$ such that

$$
\left\{\begin{array}{l}
a_{1} u_{1}=a_{2} u_{2}=\cdots=a_{h} u_{h}=w, \\
a_{1} v_{1}=a_{2} v_{2}=\cdots=a_{h} v_{h}=y,
\end{array}\right.
$$

where $u_{i} \neq v_{i}$ and $\left\{u_{1}, \ldots, u_{h}\right\}=\left\{v_{1}, \ldots, v_{h}\right\}=\left\{a_{1}, \ldots, a_{h}\right\}$. Since $\left\{u_{1}, \ldots, u_{h}\right\}=$ $\left\{v_{1}, \ldots, v_{h}\right\}$, there exist $i_{1} \neq 1, i_{2} \neq i_{1}, \ldots, i_{k} \neq i_{k-1}$ for some $k \leq h$ such that $v_{1}=u_{i_{1}}, v_{i_{1}}=u_{i_{2}}, \ldots, v_{i_{k-1}}=u_{i_{k}}$ and $v_{i_{k}}=u_{1}$. Thus

$$
\left\{\begin{array}{l}
a_{1} u_{1}=a_{i_{1}} u_{i_{1}}=\cdots=a_{i_{k}} u_{i_{k}}, \\
a_{1} u_{i_{1}}=a_{i_{1}} u_{i_{2}}=\cdots=a_{i_{k}} u_{1} .
\end{array}\right.
$$

For convenience, without loss of generality, we may assume that $i_{1}=2, i_{2}=3, \ldots, i_{k}=$ $k+1$. Then we have

$$
\left\{\begin{array}{l}
a_{1} u_{1}=a_{2} u_{2}=\cdots=a_{k+1} u_{k+1}, \\
a_{1} u_{2}=a_{2} u_{3}=\cdots=a_{k+1} u_{1} .
\end{array}\right.
$$

Thus $a_{1} u_{1} a_{i} u_{i+1}=a_{1} u_{2} a_{i} u_{i}$ for $i \leq k$ and $a_{1} u_{1} a_{k+1} u_{1}=a_{1} u_{2} a_{k+1} u_{k+1}$. Therefore, $u_{1} u_{i+1}=u_{2} u_{i}$ for $i \leq k$ and $u_{1}^{2}=u_{2} u_{k+1}$. Let $U=\left\{u_{1}, \ldots, u_{k+1}\right\}$. Then $u_{1} U=u_{2} U$. Similarly, we have $u_{1} U=\cdots=u_{k+1} U$. We claim that $a_{1}^{-1} U$ is a subgroup of $G$. In fact, for any $i, j$ with $1 \leq i, j \leq k+1$, there exists an integer $l$ such that $u_{1} u_{i}=u_{j} u_{l}$ because $u_{1} U=u_{j} U$. Thus $u_{i} u_{j}^{-1}=u_{1}^{-1} u_{l}$ and so

$$
u_{1}^{-1} u_{i} \cdot\left(u_{1}^{-1} u_{j}\right)^{-1}=u_{i} u_{j}^{-1}=u_{1}^{-1} u_{l} \in u_{1}^{-1} U
$$

Therefore, $u_{1}^{-1} U$ is a subgroup of $G$ and $U$ is a coset of the subgroup $u_{1}^{-1} U$. Now $\operatorname{Cay}(\langle U\rangle, U) \cong C_{l}\left[\bar{K}_{|U|}\right]$ is a subgraph of $\operatorname{Cay}(G, S)$ as in the theorem. This completes the proof of the theorem. 
Next we are going to characterize Cayley graphs $\operatorname{Cay}(G, S)$ for which $A_{1}^{S}$ is the alternating group or the symmetric group of degree $|S|$. To do this, we first prove the following lemma.

Lemma 3.3 Let $G$ be an Abelian group, and let $S, T$ be two Cayley subsets of $G$ such that $G=\langle S\rangle$ and $\operatorname{Cay}(G, S) \cong \operatorname{Cay}(G, T)$. If $\Gamma^{*}(x, y)=\{x y\}$ for all $x, y \in S$ and $\Gamma^{*}(u, v)=\{u v\}$ for all $u, v \in T$, then every isomorphism preserving 1 between Cay $(G, S)$ and $\operatorname{Cay}(G, T)$ induces an automorphism of $G$.

Proof: Let $S=\left\{a_{1}, a_{2}, \ldots, a_{m}\right\}$ and $T=\left\{b_{1}, b_{2}, \ldots, b_{m}\right\}$. Without loss of generality, assume that $\rho$ is an isomorphism from $\operatorname{Cay}(G, S)$ to $\operatorname{Cay}(G, T)$ such that $1 \rightarrow 1, a_{i} \rightarrow b_{i}$ for $i=1,2, \ldots, m$. Then for any $i \neq j$,

$$
\rho:\left\{a_{i} a_{j}\right\}=\Gamma^{*}\left(a_{i}, a_{j}\right) \mapsto \Gamma^{*}\left(b_{i}, b_{j}\right)=\left\{b_{i} b_{j}\right\} .
$$

We claim that $\rho$ is an automorphism of $G$. To prove this, we need only verify that for all integers $n_{1}, n_{2}, \ldots, n_{m} \geq 0$,

$$
\left(a_{1}^{n_{1}} a_{2}^{n_{2}} \cdots a_{m}^{n_{m}}\right)^{\rho}=b_{1}^{n_{1}} b_{2}^{n_{2}} \cdots b_{m}^{n_{m}},
$$

by induction on $n_{1}+n_{2}+\cdots+n_{m}$. Since

$$
\rho: \begin{cases}a_{i} \rightarrow b_{i}, & \text { for } 1 \leq i \leq m, \\ a_{i} a_{j} \rightarrow b_{i} b_{j}, & \text { for } i \neq j\end{cases}
$$

we have $\rho$ :

$$
\left\{a_{i}^{2}\right\}=\Gamma\left(a_{i}\right) \backslash\left\{a_{i} a_{j} \mid j \neq i\right\} \mapsto \Gamma\left(b_{i}\right) \backslash\left\{b_{i} b_{j} \mid j \neq i\right\}=\left\{b_{i}^{2}\right\}
$$

for all $i=1,2, \ldots, m$. In other words, (1) holds for $n_{1}+n_{2}+\cdots+n_{m} \leq 2$. Now assume inductively that the equality (1) holds for $n_{1}+n_{2}+\cdots+n_{m} \leq N$, where $N \geq 2$. Let

$$
a=\prod_{j=1}^{m} a_{j}^{n_{j}^{\prime}}, \quad \text { where } \sum_{j=1}^{m} n_{j}^{\prime}=N-1 \text {. }
$$

By the induction assumption, we have

$$
\rho:\left\{\begin{array}{l}
a \rightarrow b=\prod_{j=1}^{m} b_{j}^{n_{j}^{\prime}}, \\
a a_{i} \rightarrow b b_{i}, \quad \text { for } 1 \leq i \leq m .
\end{array}\right.
$$

Since $G$ is Abelian, for any $x \in\langle S\rangle, y \in\langle T\rangle$ and any $i \neq j$, we have $\Gamma^{*}\left(x a_{i}, x a_{j}\right)=$ $\left\{x a_{i} a_{j}\right\}$ and $\Gamma^{*}\left(y b_{i}, y b_{j}\right)=\left\{y b_{i} b_{j}\right\}$. Hence $\rho$ :

$$
\left\{\begin{array}{l}
\left\{a a_{i} a_{j}\right\}=\Gamma^{*}\left(a a_{i}, a a_{j}\right) \mapsto \Gamma^{*}\left(b b_{i}, b b_{j}\right)=\left\{b b_{i} b_{j}\right\}, \quad \text { for } 1 \leq i \neq j \leq m, \\
\left\{a a_{i}^{2}\right\}=\Gamma\left(a a_{i}\right) \backslash\left\{a a_{i} a_{j} \mid j \neq i\right\} \mapsto \Gamma\left(b b_{i}\right) \backslash\left\{b b_{i} b_{j} \mid j \neq i\right\}=\left\{b b_{i}^{2}\right\} .
\end{array}\right.
$$


Therefore, the equality (1) holds for $n_{1}+n_{2}+\cdots+n_{m}=N+1$. By induction, the equality (1) holds for all $n_{1}, n_{2}, \ldots, n_{m} \geq 0$. Hence $\rho$ is an automorphism of $G$ sending $S$ to $T$.

To prove our next theorem, we need some notation. If $a, b \in S$ and $b \neq a^{-1}$, then the product $a b$ (in $G$ ) is said to be a word of length 2 on $S$. Let $w(a b)$ be the number of all words of length 2 on $S$ which are equal to $a b$, that is, $w(a b)=\mid\{u v \mid u v=a b$ and $u, v \in S\} \mid$. For $Y \subseteq \Gamma_{2}(1)$, let $w(Y)$ be the number of all words of length 2 on $S$ which are equal to an element of $Y$, that is, $w(Y)=\sum_{y \in Y} w(y)$.

Lemma 3.4 Using the notation defined above, we have

(i) if $1 \neq a b \in \Gamma^{*}\left(u_{1}, u_{2}, \ldots, u_{i}\right)$, then $w(a b)=i$ for any $u_{1}, u_{2}, \ldots, u_{i} \in S$;

(ii) if $\left|\Gamma^{*}\left(u_{1}, \ldots, u_{i}\right)\right|=j$ then

$$
w\left(\Gamma^{*}\left(u_{1}, \ldots, u_{i}\right)\right)= \begin{cases}i j & \text { if } 1 \notin \Gamma^{*}\left(u_{1}, \ldots, u_{i}\right), \\ i(j-1) & \text { if } 1 \in \Gamma^{*}\left(u_{1}, \ldots, u_{i}\right)\end{cases}
$$

(iii) $\left|\Gamma_{2}(1)\right| \leq w\left(\Gamma_{2}(1)\right)$ and if $1 \in \Gamma^{*}(R)$ then $w\left(\Gamma_{2}(1)\right)=m^{2}-|R|$ for any $R \subseteq S$;

(iv) if $A_{1}^{S} \geq A$ lt $(|S|)$, then $1 \in \Gamma^{*}(R)$ for $R \subseteq S$ implies $R=S$.

Proof: By definition, part (i) is clear. It follows that part (ii) holds. Now let $S=$ $\left\{a_{1}, \ldots, a_{m}\right\}$. Then $\Gamma_{2}(1)=\left\{a_{i} a_{j} \mid 1 \leq i, j \leq m\right\} \backslash\{1\}$. It follows that part (iii) is true. Noting that $A_{1}^{S}$ is $(m-2)$-transitive on $S$, in particular, transitive and 2-set-transitive on $S$, part (iv) is clearly true.

Now we can prove our next result.

Theorem 3.5 Let $G$ be an Abelian group, and let $S$ be a generating subset of $G$ of size m. Let $\Gamma=\operatorname{Cay}(G, S)$, and let $A=A$ ut $\Gamma$ and $A_{1}$ the stabilizer of 1 in A. If $A_{1}^{S} \geq A l t(m)$, the alternating group of degree $m$, then one of the following holds:

(i) $S=G \backslash\{1\}$ and $\Gamma \cong K_{m+1}$;

(ii) $S=a H$ for some $H \leq G$, and $\Gamma \cong K_{m, m}$ or $C_{|G| / m}\left[\bar{K}_{m}\right]$;

(iii) $S=b H \backslash\{b\}$ for some $H \leq G, \Gamma \cong C_{|G| /(m+1)}\left[\bar{K}_{m+1}\right]-\frac{|G|}{o(b)} C_{o(b)}$;

(iv) $S=a^{L}$ for some $a \in S$ and some $L \leq \operatorname{Aut}(G, S)$, and $G \triangleleft A$;

(v) either $G$ is cyclic, or $G=Z_{n} \times B$, where $n$ is odd and $B$ is a 2-group of exponent 4 , and $\Gamma_{2}(1)=\bigcup_{u, v \in S} \Gamma^{*}(u, v) \cup \Gamma^{*}(S) \backslash\{1\}$.

Proof: First assume that $m=2$ and $S=\{a, b\}$. If $b=a^{-1}$ then $G=\langle a\rangle$ is cyclic and $\Gamma$ is a cycle of length $n:=o(a)$. Thus $A \cong D_{2 n}$, and so part (iv) holds in this case. Suppose that $b \neq a^{-1}$. If $\left|\Gamma^{*}(a, b)\right|=1$, then $a^{2} \neq b^{2}$ and so $\Gamma^{*}(a, b)=\{a b\}$. It follows from Lemma 3.3 that part (iv) holds. If $\left|\Gamma^{*}(a, b)\right|=2$ then $\Gamma^{*}(a, b)=\left\{a b=b a, a^{2}=b^{2}\right\}$. Thus $\left\{1, a^{-1} b\right\}$ is a subgroup of $G$ of order 2 , and $S=a\left\{1, a^{-1} b\right\}$ as in part (ii). Hence $\Gamma_{i}(1)=a^{i}\left\{1, a^{-1} b\right\}$ for all $i \geq 1$. Hence $\left|\Gamma_{i}(1)\right|=2$, and it follows that $\operatorname{Cay}(G, S) \cong$ $C_{|G| / 2}\left[\bar{K}_{2}\right]$.

In the following, assume that $m \geq 3$ and $S=\left\{a_{1}, a_{2}, \ldots, a_{m}\right\}$. Since $A_{1}^{S} \geq \operatorname{Alt}(m), A_{1}^{S}$ is $(m-2)$-transitive on $S$, in particular, $A_{1}^{S}$ is 2-set-transitive on $S$. By Lemma 3.1(iv), 
any element of $\Gamma_{2}(1)$ belongs to $\Gamma^{*}(R)$ for some $R \subseteq S$. Since either $\Gamma^{*}\left(a_{i}\right)=\emptyset$ or $\Gamma^{*}\left(a_{i}\right)=\left\{a_{i}^{2}\right\}$ and $a_{i}^{2} \neq a_{j} a_{k}$ for any $j, k \neq i$, there is at least one $n \in\{2, \ldots, m\}$ such that $\Gamma_{n}^{*} \geq 1$.

(1) Assume that there exists an integer $n$ with $3 \leq n \leq m-2$ such that $\Gamma_{n}^{*}=r \geq 1$. Then there are $n$ vertices $c_{1}, \ldots, c_{n} \in S$ such that $\left|\Gamma^{*}\left(c_{1}, \ldots, c_{n}\right)\right|=r$. Thus $\Gamma^{*}\left(c_{1}, \ldots, c_{n}\right)$ contains exactly $r$ elements of $\Gamma_{2}(1)$. By Lemma 3.4(ii) and (iv), $w\left(\Gamma^{*}\left(c_{1}, c_{2}, \ldots, c_{n}\right)\right)=r n$. Since $A_{1}$ is $(m-2)$-transitive on $S$, for any $n$ elements $x_{1}, \ldots, x_{n}$ of $S, w\left(\Gamma^{*}\left(x_{1}, \ldots, x_{n}\right)\right)=$ $r n$. Hence

$$
m^{2} \geq w\left(\Gamma_{2}(1)\right) \geq \sum_{x_{1}, \ldots, x_{n} \in S} w\left(\Gamma^{*}\left(x_{1}, \ldots, x_{n}\right)\right)=r n\left(\begin{array}{l}
m \\
n
\end{array}\right) .
$$

However, it is easy to see that $r n\left(\begin{array}{c}m \\ n\end{array}\right)>m^{2}$ since $3 \leq n \leq m-2$, a contradiction. Thus $\Gamma_{n}^{*}=0$ for $3 \leq n \leq m-2$.

(2) Assume that $\Gamma_{2}^{*}=\Gamma_{m-1}^{*}=0$. Then $\Gamma_{2}(1)=\left(\Gamma^{*}(S) \backslash\{1\}\right) \cup \Gamma^{*}\left(a_{1}\right) \cup \cdots \cup$ $\Gamma^{*}\left(a_{m}\right) \subseteq\left(\Gamma^{*}(S) \backslash\{1\}\right) \cup\left\{a_{1}^{2}, \ldots, a_{m}^{2}\right\}$. Thus $a_{i} a_{j} \in \Gamma^{*}(S)$ for any $a_{i} \neq a_{j}$. Since no two of $a_{1} a_{2}, \ldots, a_{1} a_{m}$ are equal, $\left|\Gamma^{*}(S)\right| \geq m-1 \geq 2$. Thus for any $i, j \neq 1$, there are integers $h, k$ such that $a_{1} a_{i}=a_{j} a_{h}$ and $a_{1} a_{j}=a_{i} a_{k}$. It follows that $a_{1}^{2}=a_{h} a_{k}$ and so $\Gamma^{*}\left(a_{1}\right)=\emptyset$. Thus $\Gamma^{*}(S) \backslash\{1\}=\Gamma_{2}(1)$. Hence every vertex in $\Gamma_{2}(1)$ is joined to all vertices in $\Gamma(1)=S$. Thus if $1 \in \Gamma^{*}(S)$ then $\operatorname{Cay}(G, S) \cong K_{m, m}$; if $1 \notin \Gamma^{*}(S)$ then $\operatorname{Cay}(G, S) \cong C_{\frac{|G|}{m}}\left[\bar{K}_{m}\right]$ where $|G|>2 m$. It follows that $a_{i} S=a_{j} S$ for any $a_{i}, a_{j} \in S$. Thus $H=a_{1}^{-1} S$ is a subgroup of $G$ and $S=a_{1} H$. This case is as in part (ii).

(3) Suppose that $\Gamma_{2}^{*}=r \geq 1$. By Lemma 3.1(iii), $r \leq 2$. If $r=2$, then since $A_{1}$ is 2 -settransitive on $S$, for any $u, v \in S,\left|\Gamma^{*}(u, v)\right|=2$ and so $\Gamma^{*}(u, v)=\left\{u v=v u, u^{2}=v^{2}\right\}$. It follows that $a_{1}^{2}=a_{2}^{2}$ and $a_{2}^{2}=a_{3}^{2}$, a contradiction. Thus $r=1$. Since $A_{1}$ is 2-set-transitive on $S,\left|\Gamma^{*}(u, v)\right|=1$ for any $u, v \in S$. Hence $\Gamma^{*}(u, v)=\{u v=v u\}$ or $\left\{u^{2}=v^{2}\right\}$. By Lemma 3.4(iv), $1 \notin \Gamma^{*}(u, v)$ and so $w\left(\Gamma^{*}(u, v)\right)=2$.

First assume that there are two elements $a, b \in S$ such that $\Gamma^{*}(a, b)=\left\{a^{2}=b^{2}\right\}$. Then $\Gamma^{*}(a)=\emptyset$ and $a b \notin \Gamma^{*}(a, b)$, so $a b=c d$ for some $c, d \in S \backslash\{a, b\}$. Thus $a b \in \Gamma^{*}\left(x_{1}, \ldots, x_{i}\right)$ for some $x_{1}, \ldots, x_{i} \in S$ where $i>2$. Since $\Gamma_{n}^{*}=0$ for $3 \leq n \leq m-2$ shown in (1), $i \geq m-1$ and so $w(a b) \geq m-1$. Thus $\Gamma_{m-1}^{*} \neq 0$ or $\Gamma_{m}^{*} \neq 0$. Since $w\left(\Gamma^{*}(u, v)\right)=2$ for all $u, v \in S$ where $u \neq v, \sum_{u, v \in S} w\left(\Gamma^{*}(u, v)\right)=2\left(\begin{array}{c}m \\ 2\end{array}\right)=m(m-1)$. If $\Gamma_{m-1}^{*}=s \neq 0$ then since $A_{1}^{S}$ is transitive on $S,\left|\Gamma^{*}(S \backslash\{u\})\right|=s$ for all $u \in S$. Thus $w\left(\Gamma^{*}(S \backslash\{u\})\right)=s(m-1)$ and so $\sum_{u \in S} w\left(\Gamma^{*}(S \backslash\{u\})\right)=m s(m-1)$. Since $1 \notin \Gamma^{*}(S \backslash\{u\})$, we have

$$
\begin{aligned}
w\left(\Gamma_{2}(1)\right) & \geq \sum_{u, v \in S} w\left(\Gamma^{*}(u, v)\right)+\sum_{u \in S} w\left(\Gamma^{*}(S \backslash\{u\})\right) \\
& =(s+1) m(m-1)>m^{2} \geq w\left(\Gamma_{2}(1)\right),
\end{aligned}
$$

a contradiction. Thus $\Gamma_{m-1}^{*}=0$, so $\Gamma_{m}^{*}=s \neq 0$ and $\Gamma_{2}(1)=\bigcup_{u, v \in S} \Gamma^{*}(u, v) \cup \Gamma^{*}(S) \backslash\{1\}$. Without loss of generality, suppose that $a=a_{1}$ and $b=a_{2}$, and let $a_{i}=o_{i} e_{i}$ such that $o_{i} \in G_{2^{\prime}}$ and $e_{i} \in G_{2}$, where $G_{2}$ is a Sylow 2-subgroup and $G_{2^{\prime}}$ is a Hall $2^{\prime}$-subgroup of $G$. Since $a_{1}^{2}=a_{2}^{2}, o_{1}=o_{2}=: o$ and $e_{1}^{2}=e_{2}^{2}$. For any $a_{i} \in S$ with $i \neq 1,2$, since $a_{1} a_{2} \in \Gamma^{*}(S)$, 
there is an $a_{j}$ such that $a_{1} a_{2}=a_{i} a_{j}$. If $j=i$ then $o_{i}^{2}=o_{1} o_{2}=o^{2}$ and $e_{i}^{2}=e_{1} e_{2}$, so $o_{i}=o$. If $j \neq i$ then since $a_{i} a_{j}=a_{1} a_{2}, a_{i} a_{j} \notin \Gamma^{*}\left(a_{i}, a_{j}\right)$. Since $\Gamma^{*}\left(a_{i}, a_{j}\right) \neq \emptyset$, we have $\Gamma^{*}\left(a_{i}, a_{j}\right)=\left\{a_{i}^{2}=a_{j}^{2}\right\}$. It follows that $o_{i}=o_{j}$ and $e_{i}^{2}=e_{j}^{2}$. Since $a_{i} a_{j}=a_{1} a_{2}=o^{2} e_{1} e_{2}$, we have $o_{i}=o$ and $e_{i} e_{j}=e_{1} e_{2}$. Thus, whether $j=i$ or not, we have $o_{i}=o$ and $e_{i}^{4}=\left(e_{i} e_{j}\right)^{2}=\left(e_{1} e_{2}\right)^{2}=e_{1}^{4}$. Hence $o_{1}=o_{2}=\cdots=o_{m}$ and $e_{1}^{4}=e_{2}^{4}=\cdots=e_{m}^{4}$. Note that $G=\langle S\rangle$, so $G_{2^{\prime}}=\langle o\rangle$ and $G_{2}=\left\langle e_{1}, e_{2}, \ldots, e_{m}\right\rangle$. If $e_{1}^{4} \neq 1$ then $G_{2}$ has only one subgroup of order 2. By [14, p. 59], $G_{2}$ is cyclic; if $e_{1}^{4}=1$ then $G_{2}$ is of exponent 4 . This case is as in part (v).

Now assume that $\Gamma^{*}(u, v)=\{u v\}$ for any $u, v \in S$ and that $G$ is not as in part (v). For any $T \subseteq S \backslash\{1\}$, by the previous paragraph, $\operatorname{Cay}(G, S) \cong \operatorname{Cay}(G, T)$ implies that $\Gamma^{*}\left(u^{\prime}, v^{\prime}\right)=\left\{u^{\prime} v^{\prime}\right\}$ for any $u^{\prime}, v^{\prime} \in T$ with $u^{\prime} \neq v^{\prime}$. By Lemma 3.3, $S$ is conjugate in $\operatorname{Aut}(G)$ to $T$ and so $S$ is a CI-subset. For any $\rho \in A_{1}$, let $b_{i}=a_{i}^{\rho}$ and $T=\left\{b_{1}, \ldots, b_{m}\right\}$. Then $\operatorname{Cay}(G, S)=\operatorname{Cay}(G, T)$. By Lemma 3.3, $\rho$ induces an automorphism of $G$. Thus $A_{1} \leq \operatorname{Aut}(G)$, so $A_{1}=\operatorname{Aut}(G, S)$ and $A=G A_{1}=G \rtimes \operatorname{Aut}(G, S)$, which is as in part (iv).

(4) Assume that $\Gamma_{2}^{*}=0$ and $\Gamma_{m-1}^{*}=r \geq 1$. Then $m \geq 4$. Since $A_{1}$ is transitive on $S$, we have $\left|\Gamma^{*}(S \backslash\{x\})\right|=r$ for every $x \in S$. If $r \geq 2$, then since $1 \notin \Gamma^{*}(S \backslash\{x\})$ for any $x \in S$,

$$
w\left(\Gamma_{2}(1)\right) \geq \sum_{x \in S} w\left(\Gamma^{*}(S \backslash\{x\})\right)=r m(m-1)>m^{2} \geq w\left(\Gamma_{2}(1)\right),
$$

a contradiction. Thus $r=1$. Let $v(x)$ be the unique element of $\Gamma^{*}(S \backslash\{x\})$. If $v\left(a_{1}\right)=a_{1}$, then for any $a_{i}$, we have $v\left(a_{i}\right)=a_{i}$ because $A_{1}$ is transitive on $S$. Thus $\operatorname{Cay}(G, S) \cong K_{m+1}$ as in part (i). Now suppose that $v\left(a_{1}\right) \neq a_{1}$. Then $v\left(a_{1}\right) \in \Gamma(x)$ for all $x \in S \backslash\left\{a_{1}\right\}$. Let $b=a_{1}^{-1} v\left(a_{1}\right)$ and $S^{*}=S \cup\{b\}$. We shall prove that $b^{-1} S^{*}$ is a subgroup of $G$. To do this, we need to prove that $b^{-1} a_{i} \cdot\left(b^{-1} a_{j}\right)^{-1} \in b^{-1} S^{*}$ for any $i \neq j$. Since $i \neq j$, we may assume that $j \neq 1$. Then $v\left(a_{1}\right) \in \Gamma\left(a_{j}\right)$ and so $v\left(a_{1}\right)=a_{j} a_{k}$ for some $a_{k} \in S$. Thus

$$
\begin{aligned}
b^{-1} a_{i} \cdot\left(b^{-1} a_{j}\right)^{-1} & =b^{-1} \cdot b \cdot a_{i} a_{j}^{-1} \\
& =b^{-1} \cdot a_{1}^{-1} v\left(a_{1}\right) \cdot a_{i} a_{j}^{-1} \\
& =b^{-1} \cdot a_{1}^{-1} a_{j} a_{k} \cdot a_{i} a_{j}^{-1} \\
& =b^{-1} a_{1}^{-1} a_{i} a_{k} .
\end{aligned}
$$

If $a_{i} a_{k} \in \Gamma\left(a_{1}\right)$, that is, $a_{i} a_{k}=a_{1} a_{k^{\prime}}$ for some $a_{k^{\prime}} \in S$, then $b^{-1} a_{i}\left(b^{-1} a_{j}\right)^{-1}=b^{-1} a_{1}^{-1} a_{i} a_{k}$ $=b^{-1} a_{k^{\prime}} \in b^{-1} S^{*}$. Hence $H:=b^{-1} S^{*}$ is a subgroup of $G$ and $S^{*}$ is a coset of $H$. Thus $S=$ $S^{*} \backslash\{b\}=b H \backslash\{b\}$, and $\operatorname{Cay}(G, S)=\operatorname{Cay}\left(G, S^{*}\right)-\operatorname{Cay}(G,\{b\}) \cong C_{\frac{|G|}{m+1}}\left[\bar{K}_{m+1}\right]-\frac{|G|}{k} C_{k}$ where $k=o(b)$, which are as in part (iii) of the theorem. Thus, in the following, we only need to prove that $a_{i} a_{k} \in \Gamma\left(a_{1}\right)$. Since $i \neq j, a_{i} a_{k} \neq a_{j} a_{k}=v\left(a_{1}\right)$. If $i \neq k$, then since $\Gamma_{n}^{*}=0$ for $2 \leq n \leq m-2, a_{i} a_{k} \in \Gamma^{*}(S) \cup \Gamma^{*}(S \backslash\{x\})$ for some $x \in S \backslash\left\{a_{1}\right\}$ and so $a_{i} a_{k} \in \Gamma\left(a_{1}\right)$. Thus we may assume that $i=k$, so $a_{i} a_{k}=a_{k}^{2}$. If $a_{k}^{2}=a_{1}^{2}$ then $a_{k}^{2} \in \Gamma\left(a_{1}\right)$. Hence suppose that $a_{k}^{2} \neq a_{1}^{2}$. Since $a_{j} a_{k}=v\left(a_{1}\right) \in \Gamma^{*}\left(S \backslash\left\{a_{1}\right\}\right)$, there exist $a_{h}, a_{l} \in S \backslash\left\{a_{j}, a_{k}\right\}$ such that $a_{j} a_{k}=a_{h} a_{l}$. If $l=h$ then $a_{h}^{2}=a_{j} a_{k}$ and so $\Gamma^{*}\left(a_{h}\right)=\emptyset$. Since $A_{1}$ is transitive on $S, \Gamma^{*}\left(a_{k}\right)=\emptyset$ and so $a_{k}^{2} \in \Gamma^{*}(S) \cup \Gamma^{*}(S \backslash\{x\})$ for some $x \in S$. 
Since $a_{k}^{2} \neq v\left(a_{1}\right)$, we have $a_{k}^{2} \in \Gamma\left(a_{1}\right)$. If $l \neq h$, then at least one of $a_{k} a_{h}$ and $a_{k} a_{l}$ does not belong to $\Gamma^{*}\left(S \backslash\left\{a_{j}\right\}\right)$, say, $a_{k} a_{h} \notin \Gamma^{*}\left(S \backslash\left\{a_{j}\right\}\right)$. Thus $a_{k} a_{h} \in \Gamma^{*}(S) \cup \Gamma^{*}(S \backslash\{x\})$ for some $x \in S \backslash\left\{a_{j}\right\}$, and so $a_{k} a_{h}=a_{j} a_{l^{\prime}}$ for some $l^{\prime}$, which, together with $a_{j} a_{k}=a_{h} a_{l}$, implies $a_{k}^{2}=a_{l} a_{l^{\prime}}$. Thus $\Gamma^{*}\left(a_{k}\right)=\emptyset$ and so $a_{k}^{2} \in \Gamma^{*}(S) \cup \Gamma^{*}(S \backslash\{x\})$ for some $x \in S$. Since $a_{k}^{2} \neq v\left(a_{1}\right), a_{k}^{2} \notin \Gamma^{*}\left(S \backslash\left\{a_{1}\right\}\right)$ and so $a_{k}^{2} \in \Gamma\left(a_{1}\right)$. This completes the proof of the theorem.

Theorem 3.5 gives an application to Babai and Frankl's question.

Corollary 3.6 Let $G$ be an elementary Abelian p-group, $p$ a prime, and $S$ a Cayley subset. Let $A=A u t \operatorname{Cay}(G, S)$. If $A_{1}^{S} \geq A l t(S)$, then $S$ is a CI-subset of $G$.

Proof: Since any subgroup of $G$ is still elementary Abelian group and each isomorphism between any two subgroups can be extended as an automorphism of $G$, we may assume that $\langle S\rangle=G$. By Theorem 3.5, Cay $(G, S)$ satisfies parts (i)-(iv). It is easy to check that $S$ is a CI-subset of $G$.

Remark By Theorem 3.5, the graphs in parts (i)-(iii) have been completely characterized. The graphs $\operatorname{Cay}(G, S)$ in part (iv) satisfies a very strong condition $\operatorname{Aut} \operatorname{Cay}(G, S) \leq$ $G \rtimes \operatorname{Aut}(G)$ 's.

\section{Finite $m$-DCI $p$-groups, $p$ a prime}

By definition, a finite group $G$ is a 1-DCI group if and only if all elements of $G$ of the same order are conjugate in $\operatorname{Aut}(G)$. Suppose that $G$ is a 1-DCI $p$-group. If $p$ is an odd prime then $G$ is homocyclic by the result of Shult [13]; if $p=2$ then by [7], $G$ is a homocyclic group or the quaternion group $Q_{8}$, or $G$ satisfies the following conditions:

(i) $G^{\prime}=\Phi(G)$ is homocyclic of rank $n$;

(ii) $G / G^{\prime}$ is of order $2^{n}$ or $2^{2 n}$;

(iii) the centre $\mathbf{Z}(G)$ of $G$ consists of the identity and all the involutions of $G$;

(iv) either $\mathbf{Z}(G)=G^{\prime}$, or $\mathbf{C}_{G}\left(G^{\prime}\right)=G^{\prime}$ with $\mathbf{Z}(G)=\Phi\left(G^{\prime}\right)$.

It is easy to see that homocyclic groups and $Q_{8}$ are 1-DCI groups, however, it is still difficult to characterize precisely 1-DCI 2-groups, see [7]. For $m \geq 2$, the problem of determining $m$-DCI groups is very different from the case $m=1$. By Lemma 2.4, we need to consider mainly Abelian $p$-groups. We first prove a property of Cayley graphs of arbitrary Abelian $p$-groups.

Proposition 4.1 Let $G$ be an Abelian p-group, $S$ a Cayley subset of $G$ such that $\langle S\rangle=G$ and $A=A$ ut $\operatorname{Cay}(G, S)$. If $p^{2} \chi\left|A_{1}\right|$ then either $S$ is a CI-subset, or $p \|\left|A_{1}\right|$ and $S$ contains a coset of some subgroup of $G$, where $A_{1}$ is the stabilizer of 1 in $A$.

Proof: Suppose that $|G|=p^{d}$. If $p \nmid\left|A_{1}\right|$, then $G$ is a Sylow $p$-subgroup of $A$. By Sylow Theorem and Theorem 2.1, $S$ is a CI-subset. Thus assume that $p \|\left|A_{1}\right|$. Let $P$ be a Sylow $p$-subgroup of $A$ containing $G$. Then $|P: G|=p$ and $P_{1} \cong Z_{p}$ where $P_{1}$ is the 
stabilizer of 1 in $P$, and so $P$ is non-Abelian, see $[15,4.4]$. Assume that $S$ is not a CI-subset of $G$. By Theorem 2.1, there is a $\tau \in \operatorname{Sym}(G)$ such that $G^{\tau}<A$ and $G^{\tau}$ is not conjugate to $G$. Let $g \in A$ such that $\left(G^{\tau}\right)^{g}<P$. Then $G^{\tau g} \neq G$ and $P \geq\left\langle G^{\tau g}, G\right\rangle>G$. Hence $P=\left\langle G^{\tau g}, G\right\rangle=G^{\tau g} G$ as $|P: G|=p$. Since any element in $G^{\tau g} \cap G$ commutes with all elements of $G^{\tau g}$ and $G$, we have $G^{\tau g} \cap G \leq \mathbf{Z}\left(\left\langle G^{\tau g}, G\right\rangle\right)=\mathbf{Z}(P)$. Further

$$
\left|G^{\tau g} \cap G\right|=\frac{\left|G^{\tau g}\right||G|}{\left|G^{\tau g} G\right|}=\frac{p^{d} \cdot p^{d}}{p^{d+1}}=p^{d-1} .
$$

Since $P$ is non-Abelian, $G^{\tau g} \cap G=\mathbf{Z}(P)$. For any $a \in \mathbf{Z}(P), P_{a}=P_{1^{a}}=P_{1}^{a}=P_{1}$, so $P_{1}$ fixes all vertices in $\mathbf{Z}(P)$. Now $\left\langle\mathbf{Z}(P), P_{1}\right\rangle$ is an Abelian subgroup of index $p$ in $P$. Hence $\left\langle\mathbf{Z}(P), P_{1}\right\rangle \triangleleft P$ and $\left\langle\mathbf{Z}(P), P_{1}\right\rangle$ has orbits $\{x \mathbf{Z}(P) \mid x \in G\}$ on $V \Gamma=G$. Thus $P_{1}$ fixes every $x \mathbf{Z}(P)$ setwise. Moreover, $P_{1}=\langle\alpha\rangle$ has an orbit $O$ on $S$ of length $p$. If $a \in O \subseteq S$, then since $P_{1}$ fixes $x \mathbf{Z}(P)$ setwise for each $x \in G, a^{\alpha} \in a \mathbf{Z}(P)$, so $a^{\alpha}=a z$ for some $z \in \mathbf{Z}(P)$. Thus $O=a^{\langle\alpha\rangle}=\left\{a, a z, a z^{2}, \ldots, a z^{p-1}\right\}=a\langle z\rangle$. Thus the proposition holds.

This result has been generalized in [8] to general abelian groups under certain conditions. The following lemma enables us to focus our attention on connected graphs.

Lemma 4.2 Assume that $G$ is a homocyclic p-group and that $S$ is a Cayley subset of $G$. If $S$ is a CI-subset of $\langle S\rangle$ and for any subset $T$ of $G, \operatorname{Cay}(\langle T\rangle, T) \cong \operatorname{Cay}(\langle S\rangle, S)$ implies $\langle T\rangle \cong\langle S\rangle$, then $S$ is a $C I$-subset of $G$.

Proof: Assume that $S$ is a CI-subset of $\langle S\rangle$ and that $T$ is a Cayley subset of $G$ such that $\operatorname{Cay}(\langle T\rangle, T) \cong \operatorname{Cay}(\langle S\rangle, S)$. Then $\langle T\rangle \cong \sigma\langle S\rangle$ for some isomorphism $\sigma$ from $\langle T\rangle$ to $\langle S\rangle$. Let $T^{\prime}=T^{\sigma}$. Then $\operatorname{Cay}\left(\langle S\rangle, T^{\prime}\right) \cong \operatorname{Cay}(\langle T\rangle, T) \cong \operatorname{Cay}(\langle S\rangle, S)$. Since $S$ is a CI-subset of $\langle S\rangle$, there is $\alpha \in \operatorname{Aut}(\langle S\rangle)$ such that $T^{\prime \sigma}=S$. Thus $\beta=\sigma \alpha$ is an isomorphism from $\langle T\rangle$ to $\langle S\rangle$ such that $T^{\beta}=\left(T^{\sigma}\right)^{\alpha}=T^{\prime \alpha}=S$. Since $G$ is a homocyclic $p$-group, it is easy to show that every isomorphism between any two isomorphic subgroups of $G$ can be extended as an automorphism of $G$. Let $\rho \in \operatorname{Aut}(G)$ be an extension of $\beta$. Then $T^{\rho}=T^{\beta}=S$, so $S$ is a CI-subset of $G$.

Now we can determine $m$-DCI $p$-groups for $2 \leq m \leq p+1$.

Theorem 4.3 Let $G$ be a finite $p$-group, where $p$ is prime. Then

(1) $G$ is an $m$-DCI group for $2 \leq m \leq p-1$ if and only if $p \geq 3$ and $G$ is homocyclic;

(2) $G$ is a $p$-DCI group if and only if $G$ is elementary Abelian, cyclic, or $G=Q_{8}$;

(3) $G$ is a $(p+1)$-DCI group if and only if $G$ is elementary Abelian, or $G=Z_{4}, Q_{8}$.

\section{Proof:}

(1) By Lemmas 2.3 and 2.4, we only need to prove that homocyclic $p$-groups are $m$-DCI groups. Let $S$ be a Cayley subset of $G$ of size $m$. By [8, Theorem 1.1], $S$ is a CI-subset of $\langle S\rangle$. Thus by Lemma 4.2, $S$ is a CI-subset of $G$ and $G$ is an $m$-DCI group. 
(2) By Lemmas 2.4 and 4.2, we only need to prove that elementary Abelian $p$-groups and cyclic $p$-groups are $p$-DCI groups. By [8, Theorem 1.1], $S$ is a CI-subset of $\langle S\rangle$. Thus by Lemma 4.2, $S$ is a CI-subset of $G$ and $G$ is a $p$-DCI group.

(3) By Lemmas 2.4 and 4.2, we only need to prove that elementary Abelian $p$-groups are $(p+1)$-DCI groups. Let $G=Z_{p}^{d}$ and let $S$ be a Cayley subset of $G$ such that $|S| \leq$ $p+1$. By parts (1) and (2), we only need to consider the case where $|S|=p+1$. Since $G$ is elementary Abelian, any two subgroups of $G$ of the same order are isomorphic. Thus, by Lemma 4.2, we may assume that $\langle S\rangle=G$.

If $p=2$, then by [3, Theorem 1], $G$ is a 3-DCI group. Thus assume $p \geq 3$ in the following. Suppose first that $S$ contains a coset $a H$ of some subgroup $H$ of $G$ for some $a \in S$. Since $|S|=p+1$, we have $|H|=p$ and $S=a H \cup\{b\}$ for some $b \in S$. If $b \in\langle a H\rangle$ then $G=\langle a, H\rangle$ is of order $p^{2}$, and thus by [6], $S$ is a CI-subset. If $b \notin\langle a H\rangle$ then $G=\langle a H\rangle \times\langle b\rangle \cong Z_{p}^{3}$, and thus by [4], again $S$ is a CI-subset. Suppose now that $S$ does not contain any coset of subgroups of $G$. By Theorem 3.2, $A_{1}$ is faithful on $S$. Since $|S|=p+1$, it follows that $p^{2} \chi\left|A_{1}\right|$. By Proposition 4.1, $S$ is a CI-subset and so $G$ is a $(p+1)$-DCI group. This completes the proof of the theorem.

\section{Acknowledgments}

The author gratefully acknowledges the support of an Overseas Postgraduate Research Scholarship of the Australian Department of Education, Employment and Training and a University Postgraduate Award from the University of Western Australia.

\section{References}

1. L. Babai, "Isomorphism problem for a class of point-symmetric structures," Acta Math. Acad. Sci. Hungar. 29 (1977), 329-336.

2. L. Babai and P. Frankl, "Isomorphisms of Cayley graphs I," Colloq. Math. Soc. J. Bolyai 18 (Combinatorics, Keszthely, 1976), North-Holland, Amsterdam, 1978, 35-52.

3. C. Delorme, O. Favaron, and M. Mahéo, "Isomorphisms of Cayley multigraphs of degree 4 on finite abelian groups," Europ. J. Combin. 13 (1992), 59-61.

4. E. Dobson, "Isomorphism problem for Cayley graph of $\mathbb{Z}_{p}^{3}$, , Disc. Math. 147 (1995), 87-94.

5. C.D. Godsil, "On the full automorphism group of a graph," Combinatorica 1 (1981), 243-256.

6. C.D. Godsil, "On Cayley graph isomorphisms," Ars Combin. 15 (1983), 231-146.

7. F. Gross, "2-automorphic 2-groups," J. Algebra 40 (1976), 348-353.

8. C.H. Li, "On isomorphisms of connected Cayley graphs," Disc. Math. 178 (1998), 109-122.

9. C.H. Li and C.E. Praeger, "On the isomorphism problem for finite Cayley graphs of bounded valency," preprint, 1997.

10. C.H. Li, C.E. Praeger, and M.Y. Xu, "Isomorphisms of finite Cayley digraphs of bounded valency," J. Combin. Theory, series, to appear.

11. L.A. Nowitz, "A non-Cayley-invariant Cayley graph of the elementary Abelian group of order 64," Disc. Math. 110 (1992), 223-228.

12. P.P. Pálfy, "Isomorphism problem for relational structures with a cyclic automorphism," Europ. J. Combin. 8 (1987), 35-43.

13. E.E. Shult, "On finite automorphic algebras,” Illinois J. Math. 13 (1969), 625-653.

14. M. Suzuki, Groups Theory II, Spring-Verlag, New York, 1982.

15. H. Wielandt, Finite Permutation Groups, Academic Press, New York, 1964.

16. M.Y. Xu, "On isomorphisms of Cayley digraphs and graphs of groups of order $p^{3}$," preprint. 